\title{
Photoperiodic regulation of the sucrose transporter StSUT4 affects the expression of circadian-regulated genes and ethylene production
}

\section{Izabela Chincinska ${ }^{1+}{ }^{,}$Konstanze Gier ${ }^{1}$, Undine Krügel ${ }^{1+}$, Johannes Liesche ${ }^{1+}{ }^{,}$Hongxia He ${ }^{1+}$, Bernhard Grimm ${ }^{1}$, Frans J. M. Harren ${ }^{2}$, Simona M. Cristescu ${ }^{2}$ and Christina Kühn ${ }^{1 *}$}

${ }^{1}$ Department of Plant Physiology, Institute of Biology, Humboldt University of Berlin, Berlin, Germany

2 Department of Molecular and Laser Physics, Radboud University Nijmegen, Nijmegen, Netherlands

Edited by:

Hanjo A. Hellmann, Washington

State University, USA

Reviewed by:

Paula Suárez-López, Centre for Research in Agricultural Genomics, CSIC-IRTA-UAB-UB, Spain

Alisdair Fernie, Max Planck Institute

for Plant Physiology, Germany

*Correspondence:

Christina Kühn, Department of Plant Physiology, Institute of Biology,

Humboldt University of Berlin,

Philippstr. 13, Building 12, 10115

Berlin, Germany.

e-mail:christina.kuehn@

biologie.hu-berlin.de

${ }^{\dagger}$ Present address:

Izabela Chincinska, Department of

Plant Physiology and Biotechnology,

Faculty of Biology, University of

Gdánsk, Poland.

Undine Krügel, University of Zurich Switzerland.

Johannes Liesche, Department of Plant and Environmental Biology,

University of Copenhagen,

Denmark

Hongxia He, Agro-Biotechnology

Research Institute, Jilin Academy of

Agricultural Sciences, Changchun,

China.
Several recent publications reported different subcellular localization of the sucrose transporters belonging to the SUT4 subfamily. The physiological function of the SUT4 sucrose transporters requires clarification, because down-regulation of the members of the SUT4 clade had different effects in rice, poplar, and potato. Here, we provide new data for the localization and function of the Solanaceous StSUT4 protein, further elucidating involvement in the onset of flowering, tuberization and in the shade avoidance syndrome of potato plants. Induction of an early flowering and a tuberization in the SUT4-inhibited potato plants correlates with increased sucrose export from leaves and increased sucrose and starch accumulation in terminal sink organs, such as developing tubers. SUT4 affects expression of the enzymes involved in gibberellin and ethylene biosynthesis, as well as the rate of ethylene biosynthesis in potato. In the SUT4-inhibited plants, the ethylene production no longer follows a diurnal rhythm. Thus it was concluded that StSUT4 controls circadian gene expression, potentially by regulating sucrose export from leaves. Furthermore, SUT4 expression affects clock-regulated genes such as StFT, StSOC1, and StCO, which might be also involved in a photoperiod-dependent tuberization. A model is proposed in which StSUT4 controls a phloem-mobile signaling molecule generated in leaves, which together with enhanced sucrose export affects developmental switches in apical meristems. SUT4 seems to link photoreceptor-perceived information about the light quality and day length with phytohormone biosynthesis and the expression of circadian-regulated genes.

Keywords: flowering, shade avoidance syndrome, sucrose transport, ethylene

\section{INTRODUCTION}

The physiological function of the members of the SUT4 family of sucrose transporters still remains unclear. Mutants or transgenic plants with reduced expression of the SUT4 genes do not show consistent phenotype in different plant species. In potato, inhibition of the StSUT4 expression by RNA interference is accompanied by early flowering and tuberization, as well as decreased sensitivity toward a far-red light enriched irradiation which might be due to increased sucrose export from transformant leaves at the end of the light period (Chincinska et al., 2008). In contrast, leaves of the ossut 2 mutant plants export less sucrose when compared to the wild type. As a consequence, sucrose, glucose, and fructose accumulate, resulting in growth retardation and reduced development of roots and grains (Atkins et al., 2011). Consistently with the observations in rice, the SUT4homologue from poplar, PtaSUT4, also affects sucrose export from source leaves. The decreased translocation of sucrose to the sink organs in PtaSUT4-repressed poplar plants results in an increased leaf-to-stem biomass ratio (Payyavula et al., 2011). Thus, StSUT4 from potato seems to inhibit sucrose efflux from leaves, whereas OsSUT2 from rice and PtaSUT4 appear to promote sucrose export from source leaves under normal conditions. It should be noted that rice and poplar employ a different strategy for phloem loading when compared to potato (Eom et al., 2012). In rice, a passive mode of phloem loading via sucrose diffusion through plasmodesmata was postulated, with involvement of the vacuolar OsSUT2 as a valve regulating sucrose flux into the phloem (Eom et al., 2012).

The SUT4 clade is heterogeneous and includes also sucrose facilitators described in Pisum sativum (Zhou et al., 2007), as well as sucrose proton co-transporters described in poplar and tomato (Weise et al., 2000; Reinders et al., 2008). Despite these 
differences, sequence homologies revealed phylogenetic similarities between the different functional classes.

It is therefore questionable, whether a general conclusion about localization and function of members of the SUT4 family can be drawn for the different plant species.

Functional diversity is also proposed after comparison of promoter elements of homologous SUT4 genes. For example, whereas OsSUT2 promoters contain hormone-related elements (Washio, 2003), Arabidopsis AtSUC4 has mainly elements related to the stress-response (Ibraheem et al., 2010). The cis-regulatory elements of the promoter regions of different members of the SUT4 subfamily differ significantly between rice, Arabidopsis, and potato (Ibraheem et al., 2010). This leads to different expression patterns of the SUT4 homologues in different plant species. These observations, together with the fact that inhibiting the SUT4 activity in different plant species leads to either no phenotype (Arabidopsis) or to opposite effects on sucrose export (rice, poplar, and potato), are strong arguments against functional similarities between different members of the SUT4 subfamily.

In the current paper, we aim to provide new insight into the localization and function of SUT4 function in potato.

As mentioned above, plants with reduced StSUT4 transcript levels exhibit an early flowering and tuberization phenotype and do not show the shade avoidance syndrome in response to increased red:far-red light ratio (Chincinska et al., 2008). StSUT4 mRNA accumulates in response to shading and this increase in transcript accumulation is not due to the enhanced transcriptional activity but rather due to increased transcript stability, as shown by treatment with the transcriptional inhibitor actinomycin D (Liesche et al., 2011). This increased transcript stability seems to be under the control of phytochrome B, because phyB antisense potato plants exhibiting constitutive shade avoidance symptoms do not show differences in StSUT4 transcript accumulation under white light or far-red light enriched conditions (Liesche et al., 2011).

A further observation made in StSUT4 mutants were altered transcript levels of enzymes involved in GA and ethylene biosynthesis. It was concluded that flowering and tuberization in potato share common pathways (Rodriguez-Falcon et al., 2006; Kühn, 2011). Indeed, flowering-related genes, e.g., StFT or StCO, are also involved in the regulation of tuberization in potato plants (Navarro et al., 2011; Gonzalez-Schain et al., 2012).

The inhibitory effect of ethylene on tuberization was described earlier (Mingo-Castel et al., 1974). Recent publications revealed a key role of the ethylene-dependent pathway also in the control of flowering and shade avoidance (Samach et al., 2000; Wuriyanghan et al., 2009). Ethylene and gibberellic acid biosynthesis pathways are suspected to trigger the symptoms of the shade avoidance syndrome in tobacco plants (Pierik et al., 2004; Stamm and Kumar, 2010). Furthermore, a recent publication revealed the involvement of ethylene in the delay of flowering in Arabidopsis via a DELLA-dependent pathway (Achard et al., 2007). Therefore, the main goal of the present studies was to elucidate the role of StSUT4 in the process of flower and tuber induction including quantification of the circadian-regulated gene transcripts and determination of the ethylene synthesizing capacity.

\section{RESULTS}

\section{SUBCELLULAR LOCALISATION OF SOLANACEAE SUT4 PROTEINS}

SUT4 members have been reported to be targeted either to the plasma membrane or to the vacuole (Table 1). Only in one proteomic report AtSUT4 has been shown to reside in the chloroplast envelope (Rolland et al., 2003). StSUT4 localization in potato was determined in the plasma membrane of sieve elements using specific affinity-purified peptide antibodies (Weise et al., 2000). The specificity of the antibody was validated in plants (Chincinska et al., 2008) and in yeast cells expressing StSUT4 in the yeast expression vector pDR196 (Figure 1A) by western blot analysis. Fractionation of the microsomal fraction of potato source leaf material into the endomembrane fraction and the plasma membrane fraction revealed two distinct bands of different sizes: one band corresponding to the full length protein of approx. $46 \mathrm{kDa}$ in the plasma membrane fraction and a second band corresponding potentially to a truncated version of the StSUT4 protein in the endomembranes fraction (Chincinska et al., 2008).

Recently, the subcellular localization of the homologous protein from Nicotiana tabacum, NtSUT4, was determined to be vacuolar, as shown by co-localization of the endocytosis marker FM4-64 in BY2 cells (Okubo-Kurihara et al., 2011). Nevertheless, NtSUT4 is able to complement a sucrose-uptake deficient yeast mutant strain which requires at least a small fraction of NtSUT4 to be localized at the plasma membrane (Okubo-Kurihara et al., 2011). Since the StSUT4-specific antibody is able to cross-react with NtSUT4, we performed subcellular fractionation of the microsomal membrane fraction of tobacco source leaves by twophase partitioning, in order to separate the plasma membrane from the endomembranes fraction (Figure 1B). As previously shown for StSUT4 in potato, the full length NtSUT4 protein is only detectable in plasma membranes and a very faint band is visible in the microsomal membrane fraction, whereas only a band of smaller molecular weight is recognized by the same antibody in the endomembranes fraction. This is a strong indication that NtSUT4 occurs in two different forms depending on its subcellular localization.

\section{StSUT4-GFP IS NOT EXCLUSIVELY LOCALIZED TO THE PLASMA MEMBRANE OF STABLY TRANSFORMED POTATO PLANTS}

StSUT4-GFP fusion constructs have been used previously for transient expression in tobacco and potato leaves by infiltration and subsequent subcellular localization (Chincinska et al., 2008). StSUT4-GFP was localized to the plasma membrane, and to membranes surrounding the nucleus, most likely the ER. The StSUT4 mRNA stability is regulated at the post-transcriptional level and is obviously stabilized under far-red light enrichment (He et al., 2008; Liesche et al., 2011). Although expressed under the constitutive 35S-promoter it is interesting to study StSUT4GFP expression in stably transformed plants in order to analyse its post-transcriptional regulation, especially during plant development. Solanum tuberosum ssp. andigena plants have been stably transformed with the same StSUT4 construct used before for transient transformation (Chincinska et al., 2008). Interestingly, StSUT4 is detectable at the plasma membrane mainly in sink leaves of young potato plantlets (Figures 2A,B). The epidermal cells expressing the StSUT4-GFP fusion construct seem to be 
Table 1 | Functional characterization of members of the SUT4 subfamily of sucrose transporters reveals a different localization than GFP fusion.

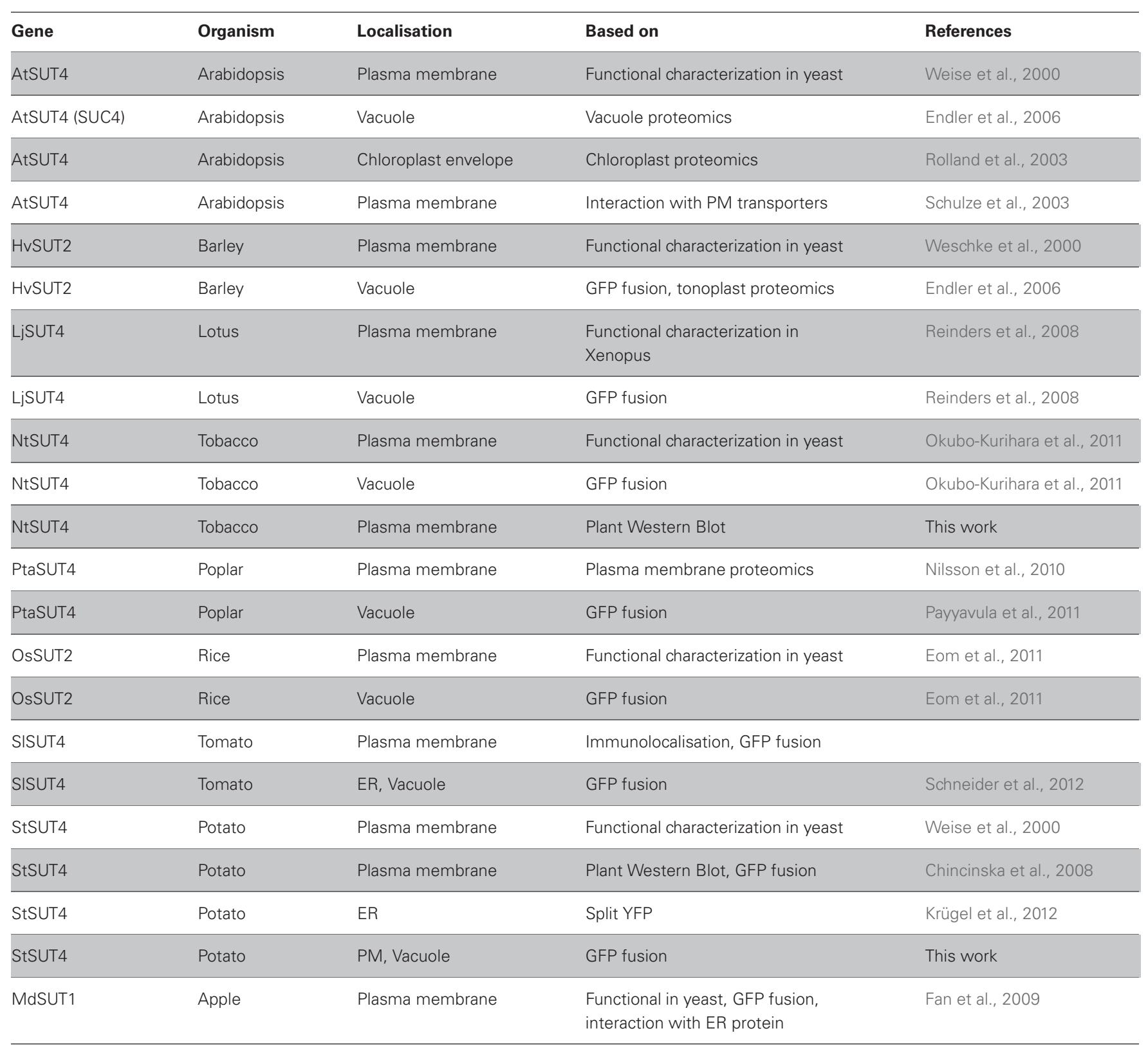

All members of the SUT4 clade are targeted to at least two different compartments.

actively undergoing cell division. Later during plant development, StSUT4-GFP expression is hardly detectable and mainly confined to the guard cells. Here, the labeling is no longer detectable at the plasma membrane. The fact that the GFP labeling in the cell periphery spares the nuclear region (Figures 2F,G) or the region of peripheral chloroplasts (Figure 2E) is a good argument for vacuolar localization. Smaller vacuolar compartments with the size of chloroplasts also reveal GFP fluorescence in mature source leaves of stably transformed potato plants (Figures 2C,D).

\section{SUT4 REDUCES SUCROSE SENSITIVITY}

The StSUT4-inhibited plants also show an increased carbon export from source leaves at the end of the light period when sucrose export arrives to its maximum (Chincinska et al., 2008). This increase in sucrose efflux correlates with significantly elevated sucrose and starch levels in sink organs such as in vitro grown microtubers. Here, the influence of exogenously supplied sucrose on tuberization in StSUT4 RNAi and wild type plants was tested by an in vitro tuberization assay. When grown in darkness, which excludes leaf carbon export effects, the induction of microtubers depended on the concentration of sucrose in the medium. Interestingly, tuber induction in StSUT4-inhibited plants was induced even in the presence of $5 \%$ or $8 \%$ of exogenous sucrose, whereas wild type potato stem cuttings start tuberization only at $10 \%$ of sucrose concentration (Figure 3 ). Thus, not only the sucrose supply to the terminal sink organs seems to 


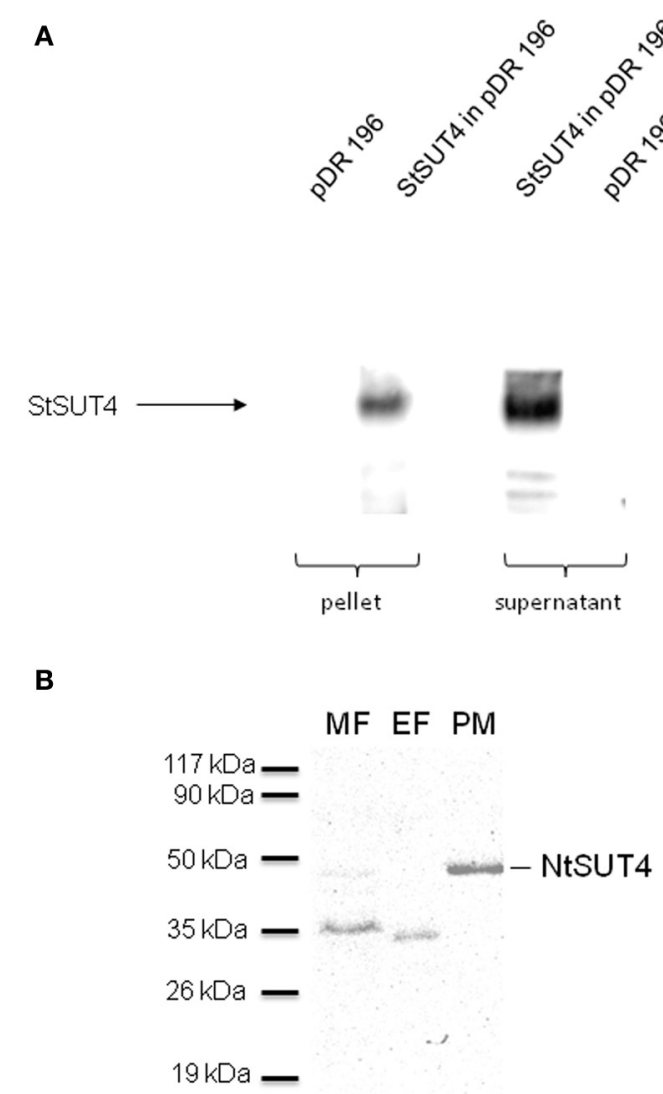

FIGURE 1 | (A) Western Blot analysis of yeast strain SUSY7 expressing StSUT4. The specificity of the StSUT4-specific polyclonal antibody generated in rabbits was tested using microsomal fractions from yeast cells expressing StSUT4 in pDR196 or the empty vector control (pDR196). The corresponding low spin pellet as well as the supernatant was loaded with Laemmli sample buffer and separated by SDS-PAGE. A band of the expected size of $46 \mathrm{kDa}$ is detectable only in yeast extracts expressing StSUT4. (B) Western blot analysis of NtSUT4 in different membrane fractions from tobacco source leaf material. The microsomal fraction (MF) was separated by two-phase partitioning into the endomembranes fraction (EF) and the plasma membrane fraction (PM) by two-phase partitioning as described earlier (Chincinska et al., 2008). $20 \mu \mathrm{g}$ of protein were loaded per lane on a $12.5 \%$ SDS gel. A band with the expected size of $46 \mathrm{kDa}$ was mainly detectable in the plasma membrane fraction. be affected by StSUT4, but also the sucrose sensitivity toward the substrate sucrose. StSUT4 seems to decrease sucrose sensitivity and sucrose efflux in wild type plants under normal growth conditions.

\section{SUT4 AFFECTS ETHYLENE PRODUCTION}

The impact of ethylene on flowering, tuberization, and shade avoidance response has been investigated further by some more recent studies (Wuriyanghan et al., 2009; Stamm and Kumar, 2010). Key enzymes of ethylene and gibberellin biosynthesis, the ACC oxidase and the GA20oxidase1 have therefore been analysed in more details and transcript levels of both key enzymes were shown to be significantly reduced in StSUT4-inhibited plants (Chincinska et al., 2008). Determination of the production of
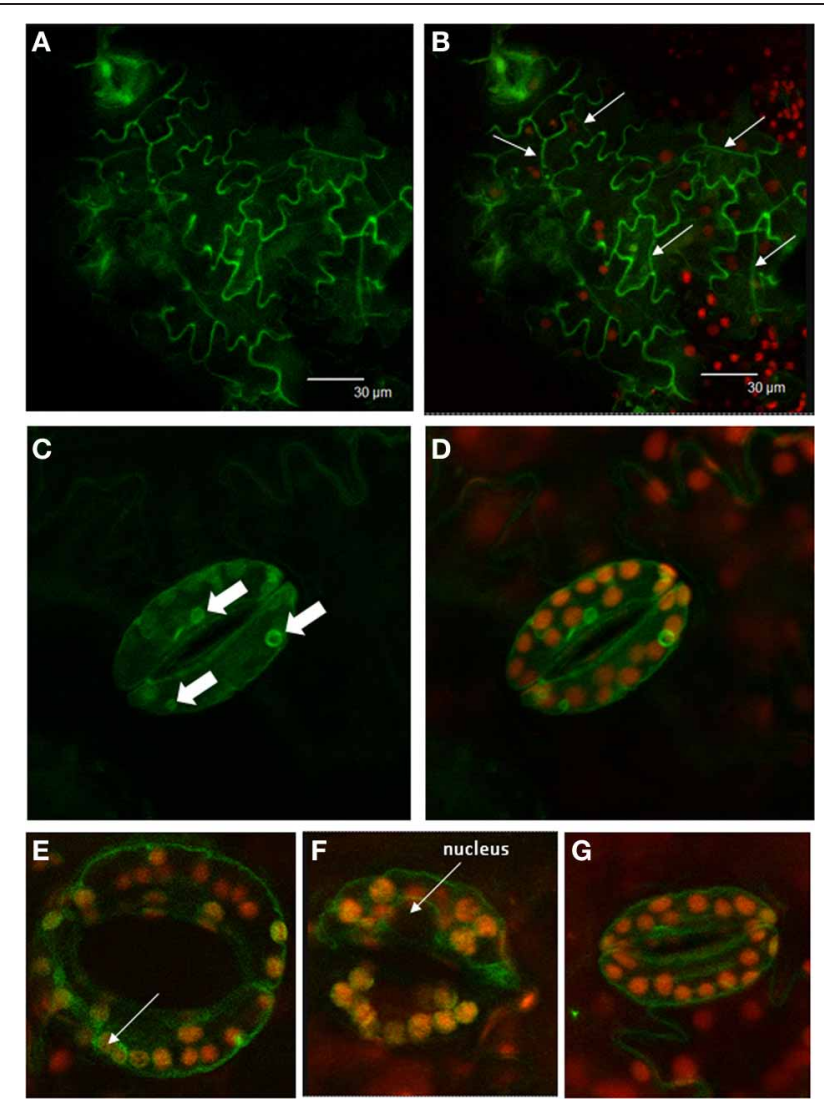

FIGURE 2 | Localization of StSUT4-GFP in stably transformed potato plants. Solanum tuberosum andigena has been transformed with StSUT4-GFP in pCK205 used previously for transient transformation (Chincinska et al., 2008). (A) StSUT4-GFP in young developing sink leaves. (B) Same picture as shown in (A) overlaid with chlorophyll autofluorescence. Arrows mark newly formed cell walls. Epidermis cells undergoing actively cell division. (C) StSUT4-GFP expression in mature source leaves is mainly restricted to guard cells. Arrows mark intracellular fluorescently labeled structures. (D) Same area as shown in (C) overlaid with chlorophyll autofluorescence indicating plastid localization. Note that GFP fluorescence is detectable outside of chloroplasts. (E-G) Guard cells of source leaves showing StSUT4-GFP fluorescence in the periphery of the cells, but sparing the region where the nucleus is localized (arrow) arguing for vacuolar localization.

the gaseous phytohormones ethylene was performed within the Trace Gas Facility of the University of Nijmegen using a portable Ethylene Sensor Sense. This device is equipped with a laser-based ethylene detector connected to a gas flow through system using air tight cuvettes. The high sensitivity of the system allows to measure ethylene amounts in the nanoliter range of a rather weak ethylene producer like potato plants. Compared to tomato plants, potato is a crop producing only very low amounts of ethylene in the range of $0.001-0.1 \mu \mathrm{l} / \mathrm{kg}$ fresh weight $\times \mathrm{h}$ (Alders, 1987; Wheeler et al., 2004).

StSUT4-inhibited potato plants of the subspecies tuberosum as well as of the strict photoperiodic subspecies andigena have been analysed using the ethylene detection system (Figures 4, A1). Whereas andigena WT plants show a diurnal rhythm of ethylene 


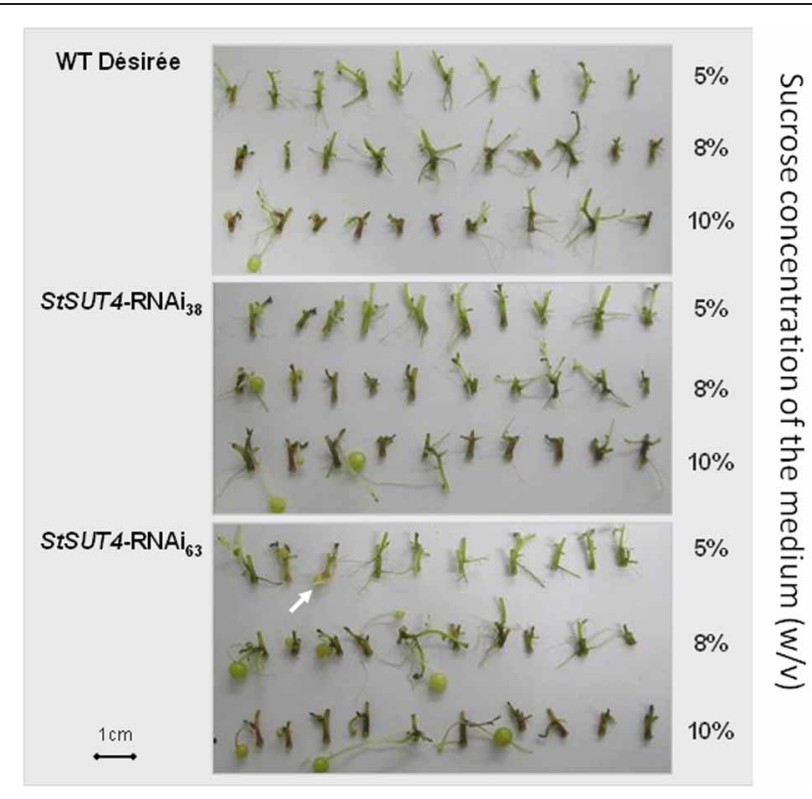

FIGURE 3 | Microtuber production in response to various sucrose concentrations in the dark. Stem cuttings from two independent transgenic lines (StSUT4-RNAi38 and StSUT4-RNAi 63) and WT potato plants from sterile tissue culture were transferred in MS-medium containing 5 , 8 , or $10 \%$ sucrose $(\mathrm{w} / \mathrm{v})$. Pictures were taken 10 days after transfer into the dark. The experiment was repeated three times independent experiments and one representative example is given. Note that microtuber production in the transgenic line StSUt4-RNAi \#63 is induced by $5 \%$ sucrose (arrow). Microtuber induction experiments were performed with Solanum tuberosum ssp. tuberosum plants of the variety Désirée. day conditions, whereas under short day conditions, when WT potato plants are induced to produce tubers, the inhibition of StSUT4 expression seems to be less important.

In order to test the hypothesis that StSUT4 expression affects flowering and tuberization induction photoperiodically, the transcript levels of known circadian-regulated genes from potato, namely StCO, StFT (or StSP6), and StSOC1, have been quantified by reverse transcription real time PCR. As shown in Figure 5 consistent differences were found for the transcript levels of these genes between wild-type and StSUT4 down regulated plants (Figure 5). The StCO transcript levels of StSUT4-inhibited plants decreased under long day conditions when compared to the wildtype plants, while the increase was observed under the short day conditions. This tendency is paralleled by the level of StSOC1. Under long day conditions, high levels of StCO mRNA during the light period (thereby enabling StCO protein synthesis) have inhibitory effect on the level of StFT mRNA in short day plants. Therefore, short day-dependent tuberization in potato plants (as well as short-day dependent flowering in SD plants) is normally inhibited under long day conditions. Since in StSUT4-inhibited plants, the level of StCO mRNA is decreased under LD conditions, the inhibitory effect on StFT mRNA levels is diminished, leading to abnormal levels of StFT mRNA under non-inductive conditions. This deregulation of flowering and tuber inducing gene expression is assumed to be responsible for tuberization of StSUT4-inhibited plants even under non-inductive long day conditions. SUT4 could therefore be a potential link between expression of StCO, StFT, and StSOC1 and day length. All phenomena are summarized in a hypothetical model shown in Figure 6.

\section{DISCUSSION HETEROGENEITY OF THE SUT4 TRANSPORTER LOCALIZATION}

Although the function of the SUT4 sucrose transporters seem to be highly diverse, many members of this phylogenetic clade seem to undergo dual targeting to the tonoplast and the plasma membrane (Table 1).

Recently, several putative tonoplast sorting signals have been described in plants, which have similarities to animal or yeast targeting motifs. Tonoplast and lysosome targeting signals include tyrosine-based YXXØ motifs (with $\emptyset$ representing a bulky amino acid residue) and acidic dileucine motifs (D/E) XXX L (L/I) in the $\mathrm{C}$-terminal or $\mathrm{N}$-terminal domain. An acidic dileucine motif in the C- or N-terminal domain seems to be necessary and sufficient for tonoplast targeting of peptide, glucose, or inositol transporters (Komarova et al., 2012). However, sorting signals such as tyrosine and dileucine based motifs can also be present in plasma membrane proteins where they are involved in the internalization by endocytosis (Irani and Russinova, 2009).

AtSUC4 from Arabidopsis, which was found in the vacuole, does not contain any of the putative sorting signals known from animals or other plants (Wolfenstetter et al., 2012). AtSUC4 follows a non-classical sorting pathway which depends on the AP-3 adaptor protein complex and which is potentially involved in the sorting to lytic vacuoles. A plant-specific role for the evolutionarily conserved AP-3 adaptor complex in mediating lytic vacuole 

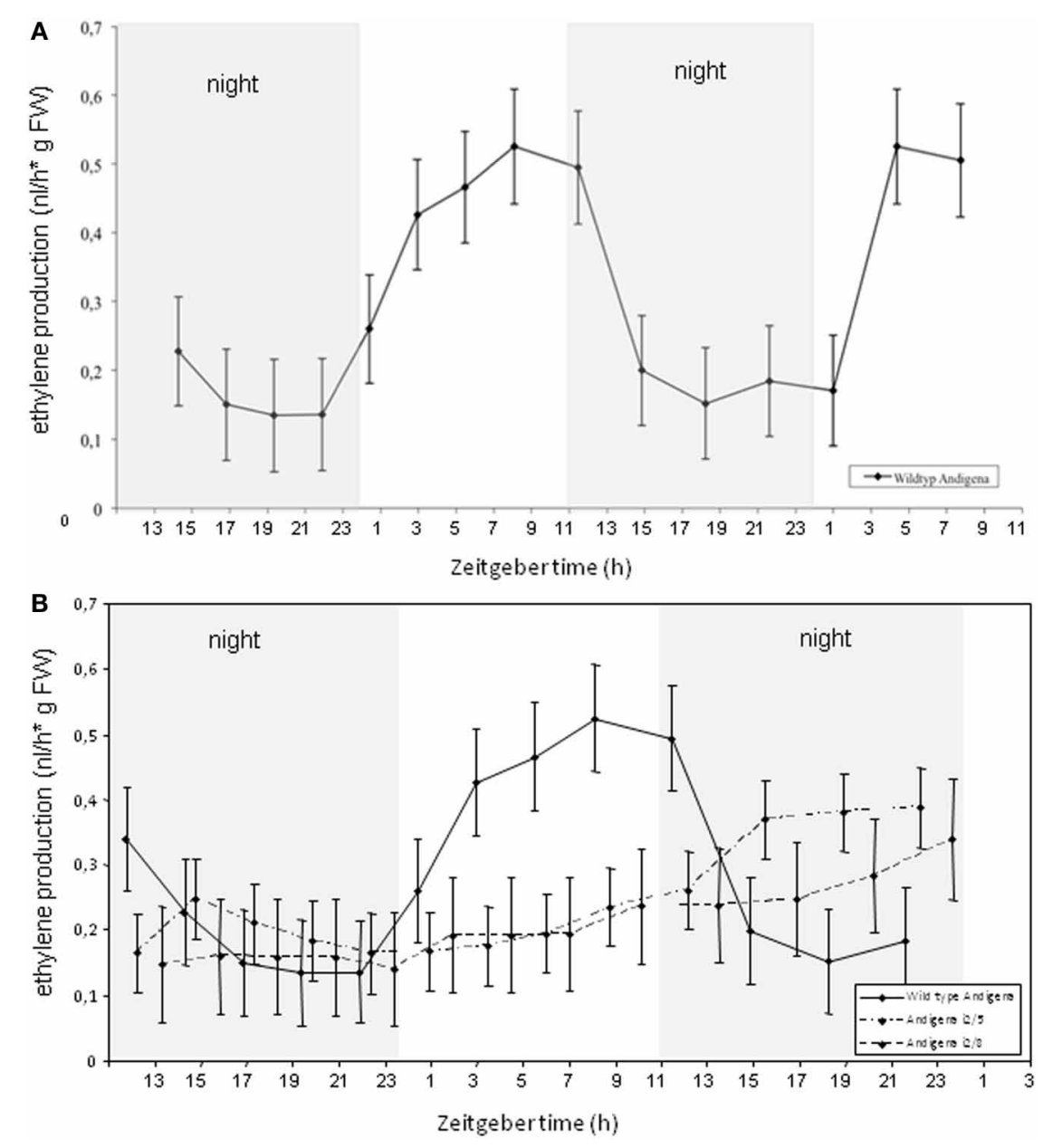

FIGURE 4 | (A) Ethylene production in Solanum tuberosum ssp. andigena plants over a 2 days period show diurnal rhythm with increasing ethylene production during day time and decreasing ethylene production during the night. Plants were grown under day neutral conditions ( $12 \mathrm{~h}$ light/12 h darkness, grey bars indicate dark periods). (B) Ethylene production in
StSUT4-RNAi plants \# i2/5 and \#i2/8 is significantly lower than in potato wild type plants and does not follow diurnal oscillation pattern. Experiments were repeated twice and a representative example is given. The ethylene production is given in nanoliters per hour per gram fresh weight. Error bars indicate the StDev. performance and transition of storage into the lytic vacuoles is suggested (Feraru et al., 2010).

Western blot analysis of potato leaves following SUT4 immunodetection suggests the presence of a truncated version of the SUT4 protein in the endomembrane fraction, whereas the full length protein is exclusively detectable in the plasma membrane fraction (Chincinska et al., 2008). It should be noted that members of the SUT4 family of sucrose transporters are degraded in lytic vacuoles to guarantee a rapid and tightly controlled turnover.

The function of StSUT4 localized at the internal membranes, as seen in guard cells of mature leaves (Figures $\mathbf{2} \mathbf{C}-\mathbf{G}$ ), remains to be elucidated. The localization at the internal membranes and small vacuoles does not resemble the targeting of SUT4 to the tonoplast, which has been reported for other plant species. Turnover of the transporter would have resulted in the complete loss of signal, as seen in the other epidermal cells of the leaf. It is possible that SUT4 is involved in the regulation of a subcellular sucrose distribution, which could be important for the osmotic pressure regulation in guard cells. However, truncation of the SUT4 protein in the endomembrane fraction suggests that the SUT4 protein undergoes degradation in lytic vacuoles. To verify the localization in vacuole-like compartments in guard cells, SUT4-GFP lines using the native promoter should be examined in order to exclude overexpression effects. Ideally, the use of an antibody that is specific for the full protein should explain where the functional SUT4 can be found within the cell.

\section{POTENTIAL SINK-SPECIFIC FUNCTION OF StSUT4 IN SUCROSE SENSING}

The physiological consequences of down-regulated SUT4 expression are diverse. The StSUT4 knock-down has different effects than inhibition or knock-out of OsSUT2, PtaSUT4, or AtSUC4. This effect could be due to the different functions of SUT4 in plants with different phloem loading strategies. Whereas poplar and rice load sucrose symplasmically, i.e., sucrose diffuses 


\section{LONG DAY}

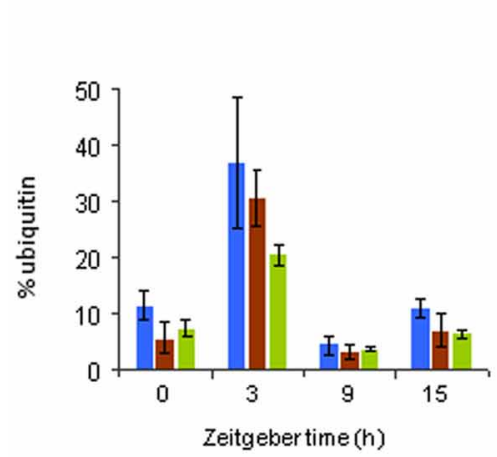

\section{SHORT DAY}

StCO

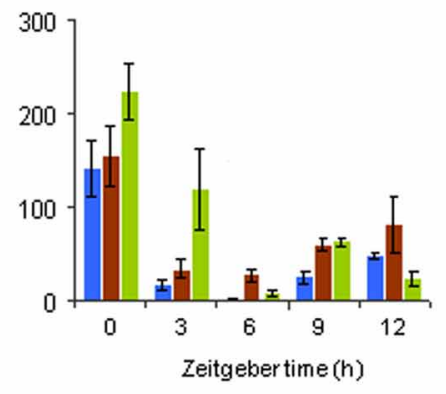

StSOC1
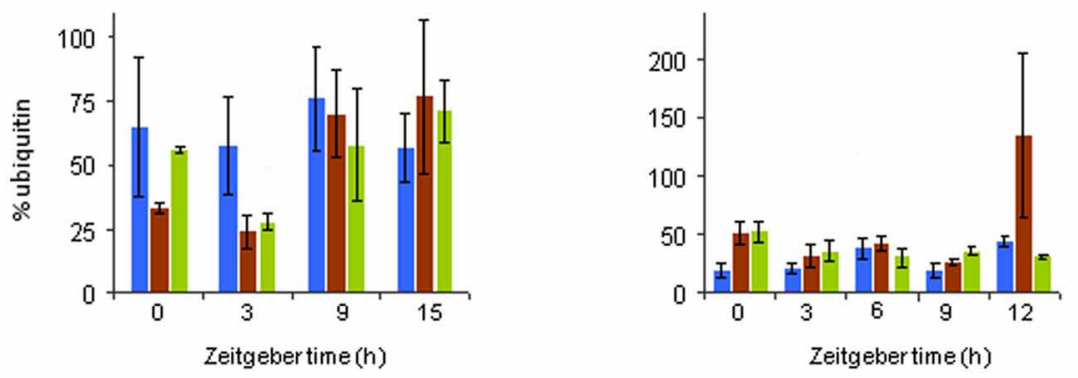

StFT

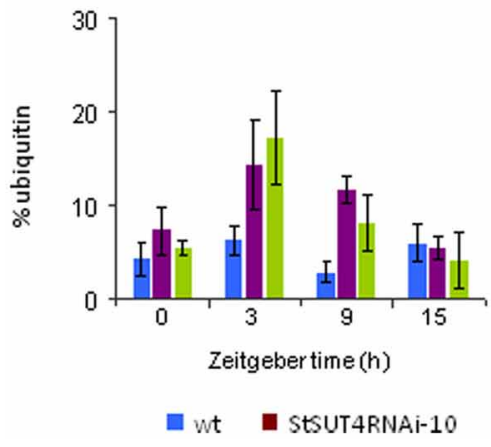

FIGURE 5 | Quantification of the transcript levels of circadian-regulated gene StFT, StSOC1, and StCO via reverse transcription real time PCR in leaves of Solanum tuberosum Désirée plants. Potato plants were grown either under short day (10 h light with a light period from 8 a.m. to $6 \mathrm{p} . \mathrm{m}$.) or long day (16 h light with a light period from 6 a.m. to 10 p.m.) conditions. StCO and StSOC1 mRNA accumulation is reduced in StSUT4-inhibited plants under short day conditions, but increased under long day conditions. StFT transcript levels are increased under both cultivation conditions, short day as well as long day conditions. Relative expression levels are given using ubiquitin mRNA as internal standard. Two technical replicates are performed from two biological replicates in each case. Experiments were repeated at least twice. One representative example is given. through plasmodesmata into the sieve element-companion cell complex, according to the cytosolic concentration potential (Fu et al., 2011; Eom et al., 2012), potato is an apoplastic loader, in which the phloem is symplasmically isolated and sucrose has to be taken up from the apoplast via membrane transporters.

Here, we report possibly a new function of StSUT4 in sink organs. The in vitro experiments (Figure 3) revealed altered sucrose-sensitivity of potato stolons, which could be indicative of a StSUT4 function in sucrose sensing. However, this effect could be also due to the increased StSUT1 expression, because StSUT1 was suggested to be negatively regulated by StSUT4 (Chincinska et al., 2008). Increased StSUT1 levels could increase the uptake of sucrose into the stolon, allowing for tuberization at lower sucrose levels.

\section{SUCROSE TRANSPORT, ETHYLENE SIGNALING, AND FLOWERING}

Involvement of sucrose transporters in ethylene signaling was already described in other plant species. Recently it was shown 


\section{Wild type StSUT4-RNAi}
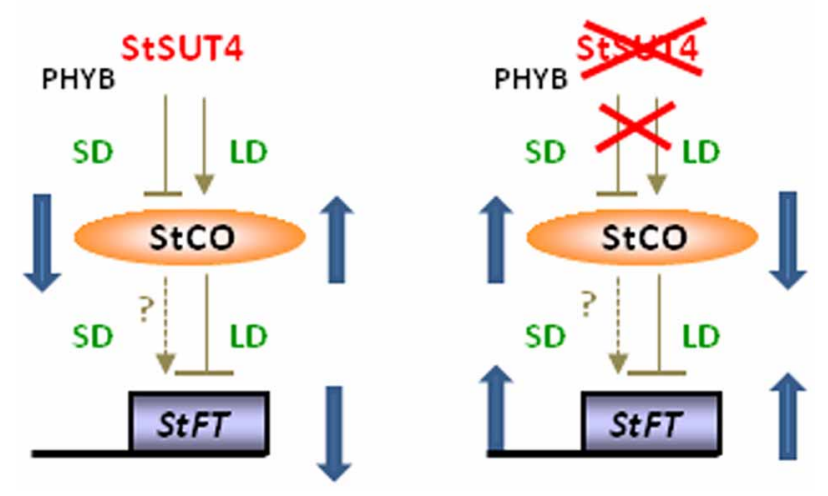

FIGURE 6 | Hypothetical model illustrating the assumed impact of the circadian-regulated gene StSUT4 on the photoperiod-dependent accumulation of StCONSTANS (StCO) and flowering locus T (StFT) transcript levels. Whereas in WT plants StSUT4 inhibits StFT accumulation under long day conditions, this photoperiod-dependent regulation via the StCONSTANS protein is deregulated in StSUT4-inhibited plants leading to increased StFT levels under both, long day and short day conditions leading to early flowering and tuberization even under non-inductive conditions.

that the regulation of sucrose transporter AtSUC1 expression by ethylene inhibits sucrose-induced anthocyanin accumulation in the presence of light (Jeong et al., 2010). Anthocyanin accumulation is positively affected by sugar and light and negatively affected by ethylene. The expression of AtSUC1 is enhanced in ethylene insensitive mutants of Arabidopsis (etr1-1, ein2-1, ein3like1) and by treatment with silver ions, which are known to inhibit ethylene perception by the ethylene receptors. AtSUC1 is assumed to play a role as an integrator for signals transmitted by sugar, light, and ethylene (Jeong et al., 2010).

In Arabidopsis, the CONSTANS protein responsible for promoting flowering, affects early targets such as SOC1 and FT, but also other target genes involved in proline or ethylene biosynthesis (Samach et al., 2000). Ethylene was shown to delay flowering, possibly by modulating DELLA activity (Achard et al., 2007). Ethylene-enhanced DELLA accumulation was assumed to delay flowering via repression of LEAFY (LFY) and SUPPRESSOR OF OVEREXPRESSION OF CONSTANS 1 (SOC1) (Achard et al., 2007). The same effect was observed in rice plants, where the overexpression of the ethylene receptor ETR2 reduced ethylene sensitivity and delayed floral transition (Wuriyanghan et al., 2009).

The role of sugars in flowering is widely accepted. In this context members of the INDETERMINATE DOMAIN (IDD) transcription factors that are involved in floral transition were described to modulate expression of sucrose transporter and sucrose metabolizing enzymes. The expression of genes encoding sucrose transporters AtSUC2, AtSUC6, AtSUC7, and AtSUC8, as well as of genes encoding sucrose synthases and invertases are affected by the expression of the IDD transcription factor IDD8 in Arabidopsis (Seo et al., 2011). IDD8 expression also affects the expression of the flowering genes FT and SOC1 and corresponding idd 8 knock out mutants show a late flowering phenotype. The IDD8 transcription factor binds directly to the SUS4 promoter and responds to photoperiodic signals. Sugar transport and metabolism is therefore tightly linked to the photoperiodic flowering.

In potato plants, StCONSTANS protein is involved in the photoperiodic control of the flowering as well as for the tuberization. StCO represses tuberization in a photoperiod-depended manner and affects the phloem-mobile StBEL5 mRNA, which promotes tuberization. Thus, StCO regulated long-distance signaling molecules in potato as well (Gonzalez-Schain et al., 2012). We suggest that StSUT4 affects the accumulation of StCO and StFT mRNA in a photoperiod-dependent manner, as it is illustrated in Figure 6. Due to the lack of StSUT4, the accumulation of StCO mRNA under SD conditions is not inhibited, leading to increased levels of StCO mRNA in StSUT4-inhibited plants. Since the inhibitory effect of StCO on StFT mRNA accumulation is assumed to occur only under LD but not under SD conditions, this increase is not accompanied by a decrease of StFT, leading to increased levels of StFT mRNA under both growth conditions.

Recently, a detailed macromolecular analysis revealed the presence of ethylene biosynthetic components in the phloem sap of Lupinus albus (Atkins et al., 2011). Since all of the known sucrose transporters from potato are phloem-localized, it can be assumed that sucrose transporter expression affects ethylene production in the phloem.

StSUT4 follows a circadian expression pattern, affects flowering, tuberization, and shade avoidance (Chincinska et al., 2008). By determination of a reduced rate of ethylene biosynthesis of StSUT4-inhibited plants, we provide first evidence for a potential link between photoperiodic flowering control and ethylene biosynthesis via sucrose transporter like protein, StSUT4.

Presently, it cannot be excluded that the effect of ethylene synthesis on the flowering behavior of potato plants involves the gibberellin-dependent pathway, since key enzymes of the gibberellin biosynthesis are also down-regulated (Chincinska et al., 2008). The important role and interplay between gibberellins and sucrose in the floral initiation has been described (Blazquez et al., 1998; King and Ben-Tal, 2001; Eriksson et al., 2006).

\section{METHODS}

\section{PLANT GROWTH CONDITIONS AND TISSUE CULTURE}

Potato plants in sterile culture were grown on 2MS-medium (MS-medium according to Murashige and Skoog, 1962 with 2\% sucrose) in tissue culture chambers at $24^{\circ} \mathrm{C}$, at $50 \%$ humidity and $1000 \mu \mathrm{mol}$ photons $\mathrm{m}^{-2} \mathrm{~s}^{-1}$ with a light/dark cycle of $16 \mathrm{~h} / 8 \mathrm{~h}$ (long day) or $10 \mathrm{~h} / 12 \mathrm{~h}$ (short day).

\section{RECOMBINANT DNA}

Generation of the StSUT4-GFP construct was described earlier (Chincinska et al., 2008). Cloning of the StSUT4 cDNA into the yeast expression vector pDR196 GW kindly provided by Doris Rentsch (Bern, Switzerland) was done using the GATEWAY technology (Invitrogen) by help of the primers: StSUT4 attB1 fw: AA AAA GCA GGC TTA ATG CCG GAG ATA GAA AGG CAT AG, 
StSUT4 attB2 rev: A GAA AGC TGG GTT TCA TGC AAA GAT CTT GGG TTT C.

\section{PLANT TRANSFORMATION}

Stable transformation of Solanum tuberosum Désirée was performed with Agrobacterium tumefaciens [strain C58C1, pGV2260; (Deblaere et al., 1985)] with small modifications according to Rocha-Sosa et al. (1989). Transformation was confirmed by PCR analysis and test for GFP fluorescence by CLSM (Leica, TCP SP2).

\section{WESTERN-BLOT ANALYSIS}

Isolation of the microsomal fraction from plant material as well as two-phase partitioning and western blotting were performed as previously described (Lemoine et al., 1996). The StSUT4-specific peptide antibody is raised against a central loop peptide of SUT4 $\left(\mathrm{NH}_{2}\right.$-CGSSHTGEEIDESSHGQEEAFLW-CONH ${ }_{2}$ ). The specificity of the affinity-purified antibody has been tested here and elsewhere (Weise et al., 2000).

\section{In vitro TUBERIZATION ASSAY}

Stem segments including at least one node of 6 weeks old sterile potato plants were prepared under sterile conditions and planted on MS medium containing 10\% sucrose. After 1 week under LD conditions in the growth chamber $\left(16 \mathrm{~h}\right.$ light, $8 \mathrm{~h}$ darkness, $24^{\circ} \mathrm{C}$ ), the scions were transferred into darkness to induce tubers. In vitro tubers were harvested after 20 days.

\section{GREENHOUSE}

Transgenic plants were amplified in tissue culture, transferred to soil and grown in a cycle of $16 \mathrm{~h}$ light $\left(22^{\circ} \mathrm{C}\right)$ and $8 \mathrm{~h}$ darkness $\left(15^{\circ} \mathrm{C}\right)$ in $60 \%$ humidity. The mean photosynthetic photon flux density (PPFD; 400-700 nm) was about $150 \mu \mathrm{mol}$ photons $\mathrm{m}^{-2} \mathrm{~s}^{-1}$ and additional illumination was provided by highpressure sodium lamps SON-T Green Power and metal halide lamps MASTER LPI-T Plus (Philips Belgium, Brussels). Emitted light from Philips SON-T Green Power has a red: far-red ratio $(660 / 730 \mathrm{~nm})$ of 2.63 and from Philips HPI-T Plus of 1.25. Both lamps are distributed equally in the green house.

\section{RNA OUANTIFICATION BY REVERSE TRANSCRIPTION REAL-TIME PCR}

RNA was isolated from different organs of greenhouse grown S. tuberosum Désirée and andigena or from leaf discs of potato plants grown in the phytochamber. RNA extraction was performed with Trisure (Bioline, Luckenwalde, Germany) or peqGold Trifast (Peqlab, Erlangen, Germany) according to the manufacturer's protocol. Reverse transcription was performed with the Qiagen Omniscript RT Kit according to the manual. Optimized conditions included using oligo(dT) primers for the initial reverse transcription reaction on approximately $1 \mu \mathrm{g}$ of total RNA after digestion with RNase-free DNase (Qiagen, Hilden, Germany).

Aliquots of $0.2 \mu \mathrm{l}$ of the $10 \mu \mathrm{l}$ RT-reaction were used for the subsequent PCR reaction in the presence of SYBR Green with HotGoldStar DNA Polymerase (Eurogentec, Seraing, Belgium) in a Rotor Gene 3000 Cycler (LTF Labortechnik, Wasserburg, Germany) using the Rotor Gene Software Version 4.6.94. The best products were obtained with the following program: denaturation at $95^{\circ} \mathrm{C}$ for $30 \mathrm{~s}$, annealing for $30 \mathrm{~s}$ at $61^{\circ} \mathrm{C}$ and elongation for $30 \mathrm{~s}$ at $72^{\circ} \mathrm{C}$, in a program of 45 cycles in $20 \mu \mathrm{l}$ reaction volume. Relative quantification of transcript amounts was always calculated in relation to the respective ubiquitin transcript level and given in $\%$ of ubiquitin. Primers were designed to obtain a 50-150 bp amplicon using Primer3 software (http://frodo.wi.mit. edu/cgi-bin/primer3/primer3_www.cgi).

Primer sequences used for reverse transcription real time PCR analysis: Ubiquitin fw: CAC CAA GCC AAA GAA GAT CA, Ubiquitin rev: TCA GCA TTA GGG CAC TCC TT; LC-SUT1 fw: TTC CAT AGC TGC TGG TGT TC; LC-SUT1 rev: TAC CAG AAA TGG GTC CAC AA; StSUT4 fw: GCT CTT GGG CTT GGA CAA GGC; StSUT4 rev: GGC TGG TGA ATT GCC TCC ACC; StFT fw: GTG GAT CCT GAT GCT CCA AG; StFT rev: TTC CTG TGG TTG CTG GGA TA; StCO fw: CTT CAA ACT CCC ATC CAC GA; StCO rev: TTG GAG TAA GCT GGG GAG GT; StSOC1 fw: TCC AGC ACG CAG GAG ATA AT; StSOC1 rev: CCA GCT TGG TTT TCA GGT TG; StGI fw: GCT TCC TCC ACA AGA TG; StGI rev: TGG ATA CCG GTT CCG TAT GA. Reverse transcription real time PCR data were corrected by calculation of the PCR efficiency individually using the LinReg PCR software.

\section{REAL-TIME MONITORING OF ETHYLENE PRODUCTION}

Plants were measured over a period of up to 2 days in 6 air tight glass cuvettes on MS medium. Empty glass cuvettes with MS medium only were subtracted from the measurements. Measurements were done in a chamber with constant temperature (Sanyo MLR-350H; Light source: Phillips, TL-D-36W/33$640 \mathrm{SLV})$. The chamber was adjusted to a light dark cycle with $11 \mathrm{~h}$ light $/ 13 \mathrm{~h}$ darkness and a temperature of $21^{\circ} \mathrm{C}$.

Ethylene production was measured with a laser-based ethylene detector (ETD-300; Sensor Sense BV; Nijmegen, Netherlands). A detailed description of the system has been given elsewhere (Cristescu et al., 2008). Briefly, the detector consists of a $\mathrm{CO}_{2}$ laser emitting radiation in the $10-\mu \mathrm{m}$ infrared wavelength region and a photoacoustic cell, in which ethylene is detected. The detector makes use of the distinct fingerprint-like absorption features of ethylene in the $\mathrm{CO}_{2}$ laser wavelength range (Cristescu et al., 2002). Inside the photoacoustic cell traces of ethylene can absorb the laser radiation; the absorbed energy is released into heat, which creates an increase in pressure inside a closed volume. By modulating the laser beam, pressure waves (i.e., sound) are generated and detected with a sensitive miniature microphone. The amplitude of the acoustic waves is directly proportional to the concentration of ethylene in the photoacoustic cell. A gas flow through system (VC-6, Sensor Sense BV; Nijmegen, Netherlands) allowed automated sampling of ethylene production under a stop-and-flow mode. Ethylene production of 6 cuvettes was accumulated during $3 \mathrm{~h}$ and then was flushed into the detector for $30 \mathrm{~min}$ at a flow rate of $3 \mathrm{l} / \mathrm{h}$. A scrubber with $\mathrm{KOH}$ (moist pellets) was used to reduce the $\mathrm{CO}_{2}$ concentration to less than $1 \mathrm{ppm}$, and a tube with $\mathrm{CaCl}_{2}$ (granules) was placed directly after this scrubber in order to decrease the water content in the gas flow. The ethylene production by the potato plants was expressed in nanoliters per hour per gram (fresh weight). Each experiment was repeated twice, and representative data are shown below. 


\section{ACKNOWLEDGMENTS}

We are very thankful for critical reading of the manuscript by Enrico Martinoia and English correction by Pawel Brzezowski. We gratefully acknowledge the support by the EU-FP6Infrastructures-5 program, project FP6-026183 "Life Science Trace Gas Facility" for allowing ethylene measurements. We

\section{REFERENCES}

Achard, P., Baghour, M., Chapple, A., Hedden, P., Van Der Straeten, D., Genschik, P., et al. (2007). The plant stress hormone ethylene controls floral transition via DELLA-dependent regulation of floral meristem-identity genes. Proc. Natl. Acad. Sci. U.S.A. 104, 6484-6489.

Alders, A. W. C. (1987). Marine Refrigeration Manual. Rotterdam: Rotterdam Marine Chartering Agents.

Atkins, C. A., Smith, P. M., and Rodriguez-Medina, C. (2011). Macromolecules in phloem exudates-a review. Protoplasma $248,165-172$.

Blazquez, M. A., Green, R., Nilsson, O., Sussman, M. R., and Weigel, D. (1998). Gibberellins promote flowering of arabidopsis by activating the LEAFY promoter. Plant Cell 10, 791-800.

Chincinska, I. A., Liesche, J., Krügel, U., Michalska, J., Geigenberger, P., Grimm, B., et al. (2008). Sucrose transporter StSUT4 from potato affects flowering, tuberization, and shade avoidance response. Plant Physiol. 146, 515-528.

Cristescu, S. M., De Martinis, D., Te Lintel Hekkert, S., Parker, D. H., and Harren, F. J. (2002). Ethylene production by Botrytis cinerea in vitro and in tomatoes. Appl. Environ. Microbiol. 68, 5342-5350.

Cristescu, S. M., Persijn, S. T., Hekkert, S. T. L., Parker, D. H., and Harren, F. J. M. (2008). Laser-based systems for trace gas detection in life sciences. Appl. Phys. B 92, 343-349.

Deblaere, R., Bytebier, B., De Greve, H., Deboeck, F., Schell, J., Van Montagu, M., et al. (1985). Efficient octopine Ti plasmid-derived vectors for Agrobacterium-mediated gene transfer to plants. Nucleic Acids Res. $13,4777-4788$.

Endler, A., Meyer, S., Schelbert, S., Schneider, T., Weschke, W., Peters, S. W., et al. (2006). Identification of a vacuolar sucrose transporter in barley and Arabidopsis mesophyll cells by a tonoplast proteomic approach. Plant Physiol. 141, 196-207.
Eom, J. S., Cho, J. I., Reinders, A., Lee, S. W., Yoo, Y., Tuan, P. Q., et al. (2011). Impaired function of the tonoplastlocalized sucrose transporter in rice, OsSUT2, limits the transport of vacuolar reserve sucrose and affects plant growth. Plant Physiol. 157, 109-119.

Eom, J. S., Choi, S. B., Ward, J. M., and Jeon, J. S. (2012). The mechanism of phloem loading in rice (Oryza sativa). Mol. Cells 33, 431-438.

Eriksson, S., Bohlenius, H., Moritz, T., and Nilsson, O. (2006). GA4 is the active gibberellin in the regulation of LEAFY transcription and Arabidopsis floral initiation. Plant Cell 18, 2172-2181.

Fan, R. C., Peng, C. C., Xu, Y. H., Wang, X. F., Li, Y., Shang, Y., et al. (2009). Apple sucrose transporter SUT1 and sorbitol transporter SOT6 interact with cytochrome b5 to regulate their affinity for substrate sugars. Plant Physiol. 150, 1880-1901.

Feraru, E., Paciorek, T., Feraru, M. I., Zwiewka, M., De Groodt, R., De Rycke, R., et al. (2010). The AP3 beta adaptin mediates the biogenesis and function of lytic vacuoles in Arabidopsis. Plant Cell 22, 2812-2824.

Fu, Q., Cheng, L., Guo, Y., and Turgeon, R. (2011). Phloem loading strategies and water relations in trees and herbaceous plants. Plant Physiol. 157, 1518-1527.

Gonzalez-Schain, N. D., DiazMendoza, M., Zurczak, M. and Suarez-Lopez, P. (2012). Potato CONSTANS is involved in photoperiodic tuberization in a graft-transmissible manner. Plant $\mathrm{J}$. 70, 678-690

He, H., Chincinska, I., Hackel, A. Grimm, B., and Kühn, C. (2008). Phloem mobility and stability of sucrose transporter transcripts. Open Plant Sci. J. 2, 1-14.

Ibraheem, O., Botha, C. E., and Bradley, G. (2010). In silico analysis of cisacting regulatory elements in $5^{\prime}$ regulatory regions of sucrose transporter gene families in rice (Oryzo sativa Japonica) and Arabidopsis thaliana. Comput. Biol. Chem. 34, 268-283.

Irani, N. G., and Russinova, E. (2009). Receptor endocytosis and signaling

would like to thank Antje Rövert for help with reverse transcription real time PCR analysis, Benjamin Herzog for help with confocal analysis, Aleksandra Hackel for excellent technical assistance and Angelika Pötter for the care of green house plants. We also thank the reviewers for helpful comments.

in plants. Curr. Opin. Plant Biol. 12, 653-659.

Jeong, S. W., Das, P. K., Jeoung, S. C., Song, J. Y., Lee, H. K., Kim, Y. K., et al. (2010). Ethylene suppression of sugar-induced anthocyanin pigmentation in Arabidopsis. Plant Physiol. 154, 1514-1531.

King, R. W., and Ben-Tal, Y. (2001). A florigenic effect of sucrose in Fuchsia hybrida is blocked by gibberellin-induced assimilate competition. Plant Physiol. 125, 488-496.

Komarova, N. Y., Meier, S., Meier, A. Grotemeyer, M. S., and Rentsch, D. (2012). Determinants for Arabidopsis peptide transporter targeting to the tonoplast or plasma membrane. Traffic. 13, 1090-1105.

Krügel, U., He, H. X., Gier, K., Reins, J., Chincinska, I., Grimm, B., et al. (2012). The potato sucrose transporter StSUT1 interacts with a DRM-associated protein disulfide isomerase. Mol. Plant 5 , 43-62.

Kühn, C. (2011). "The role of the sucrose molecule in the induction of photoperiodic responses: flowering and tuberization in potato plants share common pathways," in The Flowering Process and Its Control in Plants: Gene Expression and Hormone Interaction, ed M.W. Yaish (Trivandrum, Kerala: Research Signpost), 71-99.

Lemoine, R., Kühn, C., Thiele, N. Delrot, S., and Frommer, W. B. (1996). Antisense inhibition of the sucrose transporter in potato: effects on amount and activity. Plant Cell Environ. 19, 1124-1131.

Liesche, J., Krügel, U., He, H. Chincinska, I., Hackel, A., and Kühn, C. (2011). Sucrose transporter regulation at the transcriptional, post-transcriptional and post-translational level. J. Plant Physiol. 168, 1426-1433.

Martinez-Garcia, J. F., Virgos-Soler, A., and Prat, S. (2002). Control of photoperiod-regulated tuberization in potato by the Arabidopsis flowering-time gene CONSTANS Proc. Natl. Acad. Sci. U.S.A. 99, 15211-15216.

Mingo-Castel, A. M., Negm, F. B., and Smith, O. E. (1974). Effect of carbon dioxide and ethylene on tuberization of isolated potato stolons cultured in vitro. Plant Physiol. 53, 798-801.

Murashige, T., and Skoog, F. (1962). A revised medium for rapid growth and bio-assays with tobacco tissue cultures. Physiol. Plant. 15, 473-497.

Navarro, C., Abelenda, J. A., Cruz-Oro, E., Cuellar, C. A., Tamaki, S., Silva, J., et al. (2011). Control of flowering and storage organ formation in potato by FLOWERING LOCUS T. Nature 478, 119-122.

Nilsson, R., Bernfur, K., Gustavsson, N., Bygdell, J., Wingsle, G., and Larsson, C. (2010). Proteomics of plasma membranes from poplar trees reveals tissue distribution of transporters, receptors, and proteins in cell wall formation. Mol. Cell Proteomics 9, 368-387.

Okubo-Kurihara, E., Higaki, T., Kurihara, Y., Kutsuna, N., Yamaguchi, J., and Hasezawa, S. (2011). Sucrose transporter NtSUT4 from tobacco BY-2 involved in plant cell shape during miniprotoplast culture. J. Plant Res. 124, 395-403.

Payyavula, R. S., Tay, K. H., Tsai, C. J., and Harding, S. A. (2011) The sucrose transporter family in Populus: the importance of a tonoplast PtaSUT4 to biomass and carbon partitioning. Plant J. 65, 757-770.

Pierik, R., Cuppens, M. L., Voesenek, L. A., and Visser, E. J. (2004) Interactions between ethylene and gibberellins in phytochromemediated shade avoidance responses in tobacco. Plant Physiol. 136, 2928-2936

Reinders, A., Sivitz, A. B., Starker, C. G., Gantt, J. S., and Ward, J. M. (2008) Functional analysis of LjSUT4, a vacuolar sucrose transporter from Lotus japonicus. Plant Mol. Biol. 68, 289-299.

Rocha-Sosa, M., Sonnewald, U., Frommer, W., Stratmann, M. Schell, J., and Willmitzer, L. (1989). Both developmental and metabolic signals activate the promoter of a class I patatin gene. $E M B O$ J. 8 23-29.

Rodriguez-Falcon, M., Bou, J., and Prat, S. (2006). Seasonal control 
of tuberization in potato: conserved elements with the flowering response. Annu. Rev. Plant Biol. 57, 151-180.

Rolland, N., Ferro, M., SeigneurinBerny, D., Garin, J., Douce, R., and Joyard, J. (2003). Proteomics of chloroplast envelope membranes. Photosyn. Res. 78, 205-230.

Rutitzky, M., Ghiglione, H. O., Cura, J. A., Casal, J. J., and Yanovsky, M. J. (2009). Comparative genomic analysis of light-regulated transcripts in the Solanaceae. BMC Genomics 10:60. doi: 10.1186/1471-2164-1060

Samach, A., Onouchi, H., Gold, S. E., Ditta, G. S., Schwarz-Sommer, Z., Yanofsky, M. F., et al. (2000). Distinct roles of CONSTANS target genes in reproductive development of Arabidopsis. Science 288, 1613-1616.

Schneider, S., Hulpke, S., Schulz, A., Yaron, I., Holl, J., Imlau, A., et al. (2012). Vacuoles release sucrose via tonoplast-localised SUC4-type transporters. Plant Biol. (Stuttg) 14, 325-336.

Schulze, W. X., Reinders, A., Ward, J., Lalonde, S., and Frommer, W. B. (2003). Interactions between co-expressed Arabidopsis sucrose transporters in the split-ubiquitin system. BMC Biochem. 4:3. doi: 10.1186/1471-2091-4-3

Seo, P. J., Ryu, J., Kang, S. K., and Park, C. M. (2011). Modulation of sugar metabolism by an INDETERMINATE DOMAIN transcription factor contributes to photoperiodic flowering in Arabidopsis. Plant J. 65, 418-429.

Stamm, P., and Kumar, P. P. (2010). The phytohormone signal network regulating elongation growth during shade avoidance. J. Exp. Bot. 61, 2889-2903.

Washio, K. (2003). Functional dissections between GAMYB and Dof transcription factors suggest a role for protein-protein associations in the gibberellin-mediated expression of the RAmylA gene in the rice aleurone. Plant. Physiol. 133, 850-863.

Weise, A., Barker, L., Kühn, C., Lalonde, S., Buschmann, H., Frommer, W. B., et al. (2000). A new subfamily of sucrose transporters, SUT4, with low affinity/high capacity localized in enucleate sieve elements of plants. Plant Cell 12, 1345-1355.

Weschke, W., Panitz, R., Sauer, N., Wang, Q., Neubohn, B.,
Weber, H., et al. (2000). Sucrose transport into barley seeds: molecular characterization of two transporters and implications for seed development and starch accumulation. Plant J. 21, 455-467.

Wheeler, R. M., Peterson, B. V., and Stutte, G. W. (2004). Ethylene production throughout growth and development of plants. HortScience 39, 1541-1545.

Wolfenstetter, S., Wirsching, P., Dotzauer, D., Schneider, S., and Sauer, N. (2012). Routes to the tonoplast: the sorting of tonoplast transporters in Arabidopsis mesophyll protoplasts. Plant Cell 24, 215-232.

Wuriyanghan, H., Zhang, B., Cao, W. H., Ma, B., Lei, G., Liu, Y. F., et al. (2009). The ethylene receptor ETR2 delays floral transition and affects starch accumulation in rice. Plant Cell 21, 1473-1494.

Zhou, Y., Qu, H., Dibley, K. E., Offler, C. E., and Patrick, J. W. (2007). A suite of sucrose transporters expressed in coats of developing legume seeds includes novel $\mathrm{pH}$-independent facilitators. Plant J. 49, 750-764.
Conflict of Interest Statement: The authors declare that the research was conducted in the absence of any commercial or financial relationships that could be construed as a potential conflict of interest.

Received: 23 October 2012; paper pending published: 15 November 2012; accepted: 04 February 2013; published online: 20 February 2013.

Citation: Chincinska I, Gier K, Krügel

$U$, Liesche J, He H, Grimm B, Harren FJM, Cristescu SM and Kühn C (2013) Photoperiodic regulation of the sucrose transporter StSUT4 affects the expression of circadian-regulated genes and ethylene production. Front. Plant Sci. 4:26. doi: 10.3389/fpls.2013.00026

This article was submitted to Frontiers in Plant Physiology, a specialty of Frontiers in Plant Science.

Copyright (c) 2013 Chincinska, Gier, Krügel, Liesche, He, Grimm, Harren, Cristescu and Kühn. This is an openaccess article distributed under the terms of the Creative Commons Attribution License, which permits use, distribution and reproduction in other forums, provided the original authors and source are credited and subject to any copyright notices concerning any third-party graphics etc. 


\section{APPENDIX}
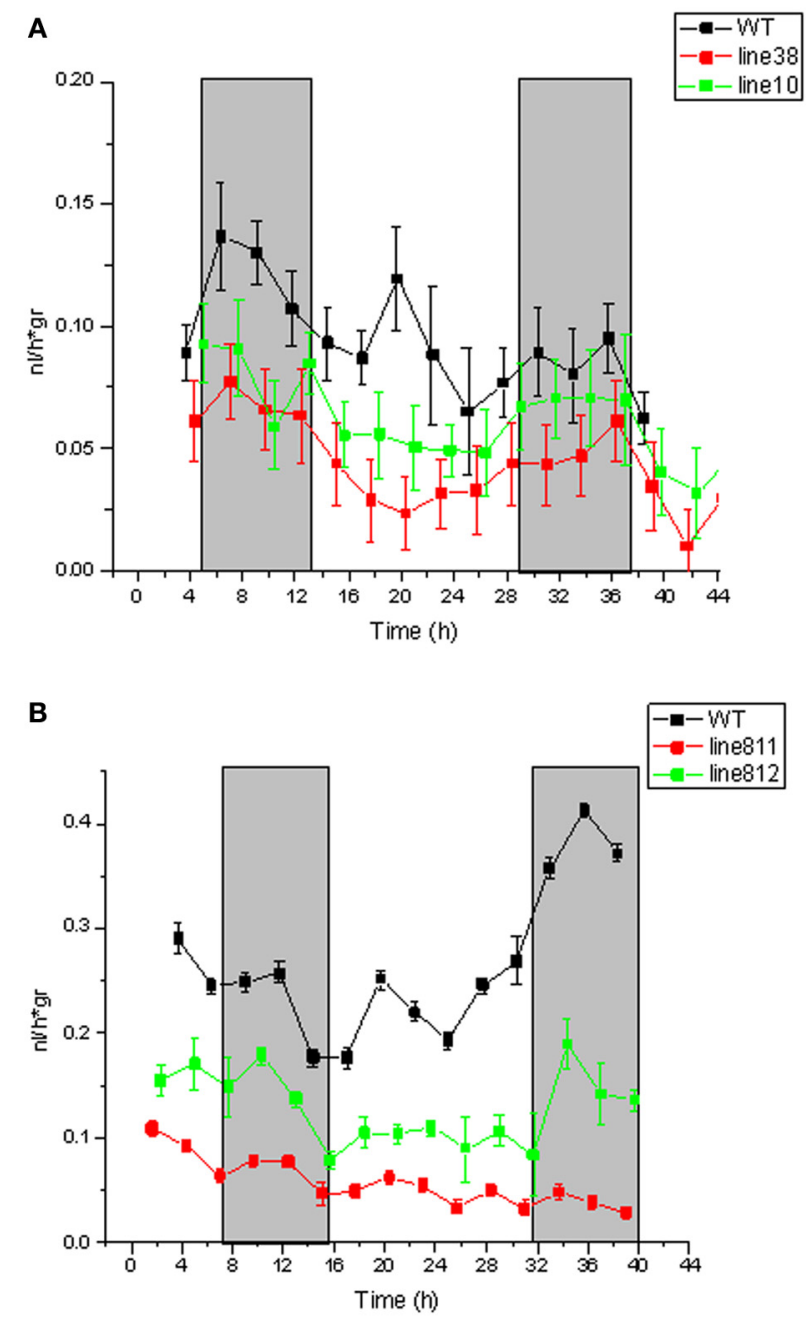

FIGURE A1 | Ethylene production in wild type Désirée and

StSUT4-RNAi potato plants. Tissue cultured plants of wild type Solanum tuberosum tuberosum Désirée and corresponding StSUT4-RNAi lines \#38, \#10 (in A) and two different plants of the transgenic line StSUT4-RNAi \#81 (in B) have been analyzed. Time is in $\mathrm{h}$ and the grey areas indicate the dark period during the experiment. Plant were grown under long day conditions (16 h light, white light of $270 \mu \mathrm{mol} / \mathrm{m}^{2} \times \mathrm{s}$, grey bars indicate dark periods). Ethylene production is given in $\mathrm{nl}$ per $\mathrm{h}$ per $\mathrm{g}$ fresh weight. Tested plants were kept 3-4 weeks in 2MS medium before ethylene measurement. Fresh weight of plants: A, WT $2.49 \mathrm{~g}$, StSUT4-RNAi \#38 $1.86 \mathrm{~g}$, StSUT4-RNAi \#10 1.84 g. B, WT 3.86 g, StSUT4-RNAi \#81.1 5.44 g, StSUT4-RNAi \#81.2 4.17 g. StDev is given. 